\title{
Stellar Parameters for a Sample of Stars with Planets
}

\author{
Luan Ghezzi ${ }^{1}$, Katia Cunha ${ }^{2,1}$, Francisco X. de Araújo ${ }^{1}$, Verne V. \\ Smith $^{2}$, Ramiro de la Reza ${ }^{1}$, and Simon Schuler ${ }^{2}$ \\ ${ }^{1}$ Observatório Nacional, Rua General José Cristino, 77, 20921-400, \\ São Cristóvão, Rio de Janeiro, RJ, Brazil; email: luan@on.br \\ ${ }^{2}$ NOAO, 950 N Cherry Ave., Tucson, AZ 85719, USA
}

\begin{abstract}
The study of chemical abundances in stars with planets is an important ingredient for the models of formation and evolution of planetary systems. In order to determine accurate abundances, it is crucial to have a reliable set of atmospheric parameters. In this work, we describe the homogeneous determination of effective temperatures, surface gravities and iron abundances for a large sample of stars with planets as well as a control sample of stars without giant planets. Our results indicate that the metallicity distribution of the stars with planets is more metal rich by $\sim 0.13$ dex than the control sample stars.
\end{abstract}

Keywords. stars: abundances, stars: atmospheres, (stars:) planetary systems: formation

\section{Introduction}

More than 400 stars with planets have been detected up to this date. One of the interesting properties of these stars concerns their metallicity distribution. Several studies (e.g., Fischer \& Valenti 2005) have confirmed the result first shown by Gonzalez (1997): stars with giant planets are systematically metal-rich (by $\sim 0.2$ dex) relative to field FGK dwarfs not known to harbor planets. Two hypotheses have been proposed to explain this excess: primordial enrichment or pollution. Current results (e.g., Fischer \& Valenti 2005; Ecuvillon et al. 2006) show that the frequency of planets increases significantly for higher metallicities, thus giving support for the primordial hypotheses. Evidence for the occurence of pollution is still ambiguous (see, e.g. Gonzalez 2006).

\section{Observations and Data Reduction}

We have observed a sample of 156 and 160 stars with and without planets, respectively. Spectra of the program stars were obtained at the MPG/ESO-2.20m telescope (La Silla, Chile) with the FEROS spectrograph (under the agreement ESO-ON). They have an almost complete spectral coverage from 3500 to $9200 \AA, \mathrm{R} \sim 48.000$ and $\mathrm{S} / \mathrm{R}>200-$ 300 (at $\sim 6300 \AA$ ). The data were reduced with the FEROS-DRS package. We note that 6 stars of this sample have already been analyzed for ${ }^{6} \mathrm{Li}$ in Ghezzi et al. (2009), showing no evidence of pollution.

\section{Determination of the Stellar Parameters}

Stellar parameters for all the target stars were derived spectroscopically and followed standard techniques. Effective temperatures were obtained from zero slopes in diagrams of $\mathrm{A}(\mathrm{Fe} \mathrm{I})$ versus $\chi$ using just lines which had $\log (E W / \lambda)<-5.00$. This limit was set to eliminate the strong lines that are sensitive to the microturbulent velocity value, 


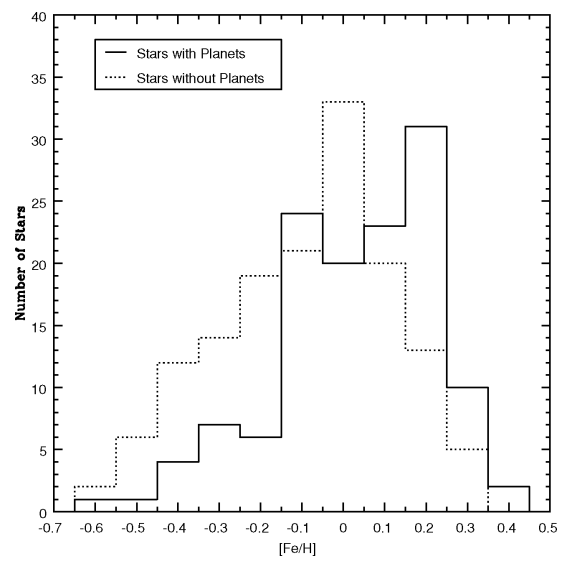

Figure 1. The metallicity distributions for a sample of 125 planet host stars (solid line) and a control sample with 149 stars without giant planets.

decoupling the $T_{\text {eff }}$ determination from the microturbulence determination. The microturbulent velocities were varied until the slopes of $\mathrm{A}(\mathrm{Fe} \mathrm{I})$ versus $\log (E W / \lambda)$ were zero. Finally, surface gravities were derived from ionization equilibrium between Fe I and Fe II species. The abundances were derived in LTE using an updated version of the spectrum synthesis code MOOG (Sneden 1973). The model atmospheres adopted in the analysis were interpolated from the ODFNEW grid of ATLAS9 models (Castelli \& Kurucz 2004).

\section{Results and Next Steps}

The average metallicities for stars with and without planets are +0.02 and -0.09 , respectively (Fig. 1). These results recover the offset between the average metallicities of the two samples $(\sim 0.13 \mathrm{dex})$ as well as the average metallicity for field FGK dwarfs usually found in the literature. The next step of this study is the homogeneous determination of the abundances of other chemical elements (such as $\mathrm{Ni}, \mathrm{Si}, \mathrm{Ti}, \mathrm{V}, \mathrm{Ca}, \mathrm{Na}, \mathrm{C}, \mathrm{N}, \mathrm{O}, \mathrm{Mg}$ and $\mathrm{Li}$ ) for the entire sample. The comparison of the abundance patterns for stars with and without planets (for instance, using the relation between chemical abundances of several elements and their condensation temperatures; see e.g., Smith, Cunha \& Lazzaro 2001 and Ecuvillon et al. 2006) will allow us to check the occurrence of the pollution process and understand better the formation and evolution of planetary systems.

\section{References}

Castelli, F. \& Kurucz, R. L. 2004, in: N. Piskunov (eds.) Modelling of Stellar Atmospheres, Proc.

IAU Symposium No 210 eds., poster A20 (astro-ph/0405087)

Smith, V. V., Cunha, K. \& Lazzaro, D. 2001, AJ, 121, 3207

Ecuvillon, A., Israelian, G., Santos, N. C., Mayor, M., \& Gilli, G. 2006, A\&GA, 449, 809

Fischer, D. A. \& Valenti, J. 2005, ApJ, 622, 1102

Ghezzi, L., Cunha, K., Smith, V. V., Margheim, S., Schuler, S., de Araújo, F.X., \& de la Reza, R. 2009, ApJ, 698, 451

Gonzalez, G. 1997, MNRAS, 285, 403

Gonzalez, G. 2006, PASP, 118, 1494

Smith, V. V., Cunha, K., \& Lazzaro, D. 2001, AJ, 121, 3207

Sneden, C. 1973, Ph.D. thesis, University of Texas, Austin 